\title{
Numerical Solution for a FG Cylinder Problem Using Finite-Difference Method
}

\author{
Daoud S. Mashat \\ Department of Mathematics, Faculty of Science, King AbdulAziz University, Jeddah, Saudi Arabia \\ E-mail: dmashat@kau.edu.sa \\ Received August 28, 2010; revised November 24, 2010; accepted November 28, 2010
}

\begin{abstract}
A refined finite-difference approach is presented to solve the thermoelastic problem of functionally graded cylinders. Material properties of the present cylinder are assumed to be graded in the radial direction according to a power-law distribution in terms of the volume fractions of the metal and ceramic constituents. The governing second-order differential equations are derived from the motion and the heat-conduction equations. Numerical results for dimensionless temperature, radial displacement, mechanical stresses and electromagnetic stress are distributed along the radial directions. The effects of time parameter and the functionally graded coefficient are investigated.
\end{abstract}

Keywords: Functionally Graded, Annular Cylinder, Electro-Magneto-Thermoelastic Field

\section{Introduction}

Magneto-thermoelastic functionally graded material is a kind of magneto-thermoelastic material with material composition and properties varying continuously along certain directions. It is the composite material intentionally designed so that they possess desirable properties for some specific applications. The advantage of this new kind of materials can improve the reliability of life span of magnetic devices. Recently, there has been growing interest in materials deliberately fabricated so that their electric, magnetic, thermal and mechanical properties vary continuously in space on the macroscopic scale. This research subject is so new that only a few results can be found in the literatures.

In recent years the theory of magneto-thermo-elasticity that deals with the interactions among strain, temperature and electromagnetic fields has drawn the attention of many researchers. This theory is extensive uses in diverse field such as geophysics for understanding the effect of the Earth's magnetic field on seismic waves, damping of acoustic waves in a magnetic field, and emissions of electromagnetic radiations from nuclear devices. It is also developed a highly sensitive superconducting magnetometer, electrical power engineering and optics.

In the field of magneto-elasticity or magneto-thermoelasticity, many studies have been conducted on an analytical treatment of an interaction between elastic, elec- tromagnetic, and temperature fields (e.g., Paria [1], Wang et al. [2], Wang and Dai [3], Wang and Dong [4], Banerjee and Roychoudhuri [5]).

The magneto-thermoelastic problem of functionally graded material (FGM) hollow structures subjected to mechanical loads is considered by Dai and Fu [6]. Hou and Leung [7] analyzed the plane strain dynamic problem of a magneto-electro-elastic hollow cylinder by virtue of the separation of variables, orthogonal expansion technique and the interpolation method. Buchanan [8] considered the free vibration problem of an infinite magneto-electro-elastic cylinder. The frequency behaviour of clamped-clamped magneto-electro-elastic cylindrical shells is analysed by Annigeri et al. [9] using the semi-analytical finite element approach. Hou et al. [10] studied the transient responses of a special non-homogeneous magneto-electro-elastic hollow cylinder for axisymmetric plane strain problem. The magnetother-moelastic problem in non-homogeneous isotropic cylinder in a primary magnetic field is discussed by Abd-El-Salam et $a l$. [11]. The dynamic and quasi-static behaviors of magneto-thermo-elastic stresses in a conducting hollow circular cylinder subjected to an arbitrary variation of magnetic field are investigated by Higuchi et al. [12].

Bhangale and Ganesan [13] studied the free vibration on FGM magneto-electro-elastic cylindrical shells using a series solution. Kong et al. [14] presented an analytical method to investigate thermo-magneto-elastic stresses 
and perturbation of the magnetic field vector in a conducting non-homogeneous hollow cylinder under thermal shock. Wang and Dai [15] presented magneto-thermodynamic stresses and perturbation of magnetic field vector in an orthotropic thermoelastic cylinder. Dai and Wang [16] presented an analytical solution for magneto-thermo-electro-elastic problems of a piezoelectric hollow cylinder placed in an axial magnetic field subjected to arbitrary thermal shock, mechanical load and transient electric excitation.

The objective of this investigation is to generate displacement, stresses, temperature, and magnetic field in a FGM annular cylinder. Material properties of the present cylinder are assumed to be graded in the radial direction according to a power-law distribution in terms of the volume fractions of the metal and ceramic constituents. The governing motion and the heat-conduction equations are obtained in conservation forms and solved numerically using finite difference method. A refined finitedifference approach is presented to solve the present problem. Numerical results for the variation of temperature, displacement and stresses are presented for a metalceramic FG cylinder. To make the study reasonably, temperature, displacement, and stresses are given for different homogenization schemes and exponents in the power-law that describes along-the-thickness variation of the constituents. The effects of many parameters are in-

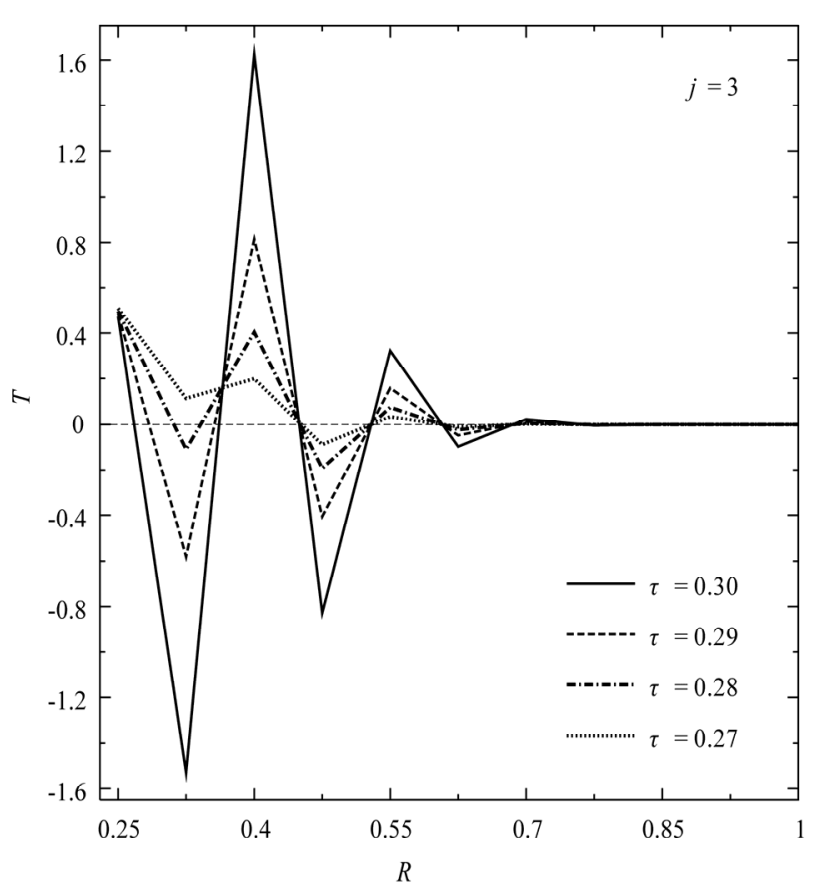

(a) vestigated.

\section{Mathematical Model}

Let us consider an annular cylinder of outer radius $r=b$, inner radius $r=a$, and made of a functionally graded material. The cylindrical coordinates system $(r, \theta, z)$ for the axially symmetric problem is used with $z$-axis coinciding with the axis of the cylinder. We have only the radial displacement $u_{r}$ which is independent of $\theta$ and $z$. The cylinder is placed in a constant primary magnetic field $H_{0}$ as shown in Figure 1. In a generalized plane strain, we suppose that the planes perpendicular to the $z$-axis and $u_{r}$ is a function of the radial direction $r$ and time $t$ only. The Cauchy's relations are

$$
e_{r r}=\frac{\partial u_{r}}{\partial r}, \quad e_{\theta \theta}=\frac{u_{r}}{r}, \quad e_{z z}=e_{z r}=e_{r \theta}=e_{z \theta}=0,
$$

where $e_{i j}$ are the strain components. The mechanical stress components $\sigma_{r r}$ and $\sigma_{\theta \theta}$, and Maxwell's electromagnetic stress component $\bar{\sigma}_{r r}$ are given, respectively, by

$$
\left.\begin{array}{c}
\sigma_{r r}=\lambda(r)\left(e_{r r}+e_{\theta \theta}\right)+2 \mu(r) e_{r r}-\gamma(r) \bar{T}, \\
\sigma_{\theta \theta}=\lambda(r)\left(e_{r r}+e_{\theta \theta}\right)+2 \mu(r) e_{\theta \theta}-\gamma(r) \bar{T}, \\
\bar{\sigma}_{r r}=\eta(r) H_{0}^{2}\left(e_{r r}+e_{\theta \theta}\right) .
\end{array}\right\}
$$

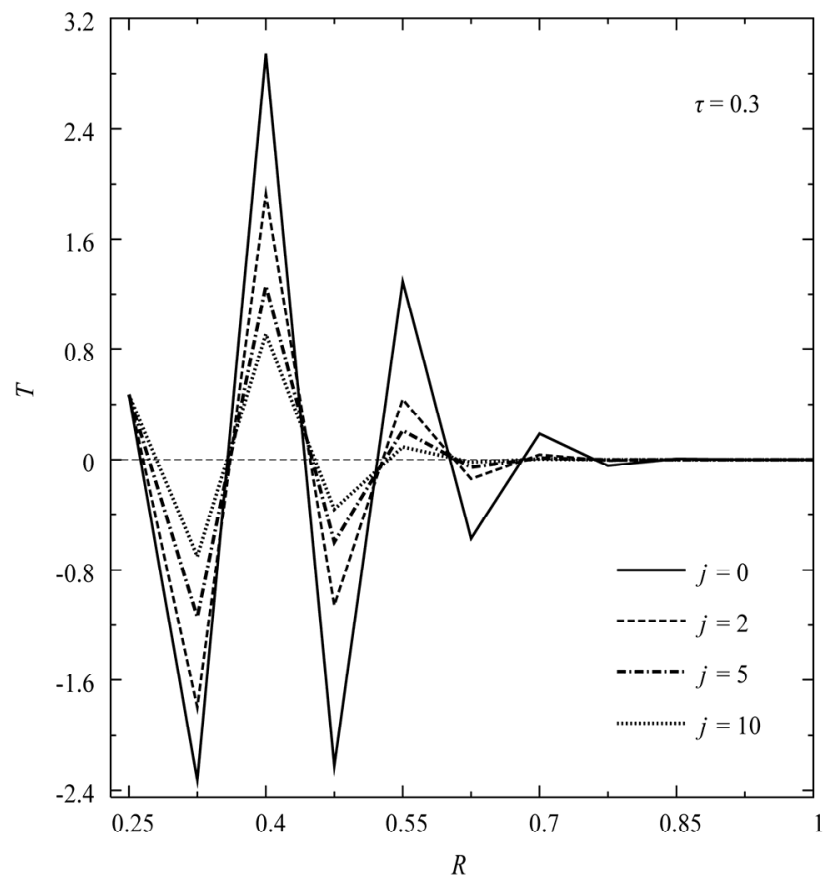

(b)

Figure 1. Temperature distribution through the radial direction of the FGM annular cylinder at (a) different times, and (b) for different values the gradation exponent. 
where $T$ is the absolute temperature, $\lambda$ and $\mu$ Lamé's coefficients, $\eta$ the magnetic permeability, $\gamma=(3 \lambda+2 \mu) \alpha$ the stress-temperature modulus, in which $\alpha$ is the linear thermal expansion, and $H_{0}$ is the primary magnetic field.

The magneto-elasto-dynamic equation in the radial direction of the FG annular cylinder is given by

$$
\frac{\partial \sigma_{r r}}{\partial r}+\frac{1}{r}\left(\sigma_{r r}-\sigma_{\theta \theta}\right)+\frac{\partial \bar{\sigma}_{r r}}{\partial r}=\rho(r) \frac{\partial^{2} u_{r}}{\partial t^{2}},
$$

where $\rho$ is the material density of the cylinder. The heat conduction equation in the absence of heat source can be written in the form [17]

$$
\left[\kappa(r)\left(\frac{\partial^{2}}{\partial r^{2}}+\frac{1}{r} \frac{\partial}{\partial r}\right)-C(r) \frac{\partial^{2}}{\partial t^{2}}\right] T_{1}=\gamma(r) T_{0} \frac{\partial}{\partial t}\left(\frac{\partial}{\partial r}+\frac{1}{r}\right) u_{r},
$$

where $T_{0}$ is the reference temperature, $C=\rho c_{v}$ the specific heat at constant volume, and $\kappa$ is the thermal conductivity.

The material properties like $\lambda, \mu, \eta, \gamma, \rho, c$, and $\kappa$ of the FGM cylinder are assumed to be function of the volume fraction of the constituent materials. The functionally graded between the physical properties and the radial direction $r$ for ceramic and metal FG cylinder is given by

$$
P(r)=\left(P_{m}-P_{c}\right)\left(1-\frac{r-a}{b-a}\right)^{j}+P_{c},
$$

where $P_{c}$ and $P_{m}$ are the corresponding properties of ceramic and metal, respectively, and $j$ is the volume fraction exponent which takes values greater than or equal to zero. The value of $j$ equal to zero represents a fully metal annular cylinder. The above power-law assumption reflects a simple rule of mixtures used to obtain the effective properties of the ceramic-metal FGM annular cylinder. The rule of mixtures applies only to the radial direction. Note that the volume fraction of the metal is high near the inner surface of the cylinder, and that of ceramic high near the outer surface. In addition, Equation (3) indicates that the inner surface of the annular cylinder is metal-rich whereas its outer surface is ceramic-rich. The density and other physical components of the annular cylinder according to the power law, and the power-law exponent may be varied to obtain different distributions of the component materials through the radial direction of the cylinder.

\section{Solution of the Problem}

Introducing the following dimensionless variables may be simplifying the solving process:

$$
\begin{aligned}
& R=\frac{r}{b}, A=\frac{a}{b}, \tau=\frac{t}{b}, T=\frac{\bar{T}(r, t)}{T_{0}}, U=\frac{u_{r}(r, t)}{b T_{0}}, \\
& \left(\sigma_{11}, \sigma_{22}\right)=\frac{1}{T_{0}}\left(\sigma_{r r}, \sigma_{\theta \theta}\right), \quad \Sigma=\frac{\bar{\sigma}_{r r}}{H_{0}^{2} T_{0}},
\end{aligned}
$$

where $\tau$ is dimensionless time and $\alpha_{0}$ is the linear thermal expansion constant. The effect of material properties variation of the FG cylinder can be taken into account in Equations (3) and (4) using Equation (5). The substitution of Equations (1) and (2) into Equations (3) and (4) with the aid of the dimensionless variables given in Equation (6) produces the governing equations for the FG cylinder as follows:

$$
\begin{aligned}
& \beta^{*}\left[R^{2} \frac{\partial^{2} U}{\partial R^{2}}+R \frac{\partial U}{\partial R}-U\right]+\left(\beta_{m}-\beta_{c}\right) R^{2} V^{\prime}(R) \frac{\partial U}{\partial R} \\
& +\zeta R V^{\prime}(R) U-\gamma^{*} R^{2} \frac{\partial T}{\partial R}-\left(\gamma_{m}-\gamma_{c}\right) V^{\prime}(R) R^{2} T \\
& =\rho^{*} R^{2} \frac{\partial^{2} U}{\partial \tau^{2}}, \\
& {\left[\varepsilon_{1}\left(\frac{\partial^{2}}{\partial R^{2}}+\frac{1}{R} \frac{\partial}{\partial R}\right)-\frac{\partial^{2}}{\partial \tau^{2}}\right] T=\varepsilon_{2} \frac{\partial}{\partial \tau}\left(\frac{\partial}{\partial R}+\frac{1}{R}\right) U,}
\end{aligned}
$$

where

$$
\begin{aligned}
& \beta_{m}=\eta_{m} H_{0}^{2}+2 \mu_{m}+\lambda_{m}, \beta_{c}=\eta_{c} H_{0}^{2}+2 \mu_{c}+\lambda_{c}, \\
& \zeta=\left(\eta_{m}-\eta_{c}\right) H_{0}^{2}+\lambda_{m}-\lambda_{c}, V(R)=\left(\frac{R-1}{A-1}\right)^{j}, \\
& \varepsilon_{1}=\frac{\kappa^{*}}{C^{*}}, \quad \varepsilon_{2}=\frac{\gamma^{*} T_{0}}{C^{*}},
\end{aligned}
$$

where the letter $\xi$ in the expression

$$
\xi^{*}=\left(\xi_{m}-\xi_{c}\right) V(R)+\xi_{c} .
$$

is given instead of the parameters $\beta, \gamma, \kappa, C$ or $\rho$. Note that, the prime (') in Equation (7) denotes differentiation with respect to $R$. Also, $\lambda_{p}, \mu_{p}, \gamma_{p}, \kappa_{p}$, $C_{p}, \eta_{p}$ and $\rho_{p}(p=m$ or $c)$ are Lamé's constants, thermal modulus, thermal conductivity, specific heat, magnetic permeability, and density of the homogeneous metal or ceramic material, respectively, and the parameter $\xi$ in Equation (10) may be used to represent one of these constants.

The dimensionless stresses induced by the temperature $T$ and the electromagnetic stress are related to the dimensionless radial displacement $U$ by

$$
\begin{aligned}
& \sigma_{11}=\lambda^{*}\left(\frac{\partial U}{\partial R}+\frac{U}{R}\right)+2 \mu^{*} \frac{\partial U}{\partial R}-\gamma^{*} T, \\
& \sigma_{22}=\lambda^{*}\left(\frac{\partial U}{\partial R}+\frac{U}{R}\right)+2 \mu^{*} \frac{U}{R}-\gamma^{*} T,
\end{aligned}
$$




$$
\Sigma=\eta^{*}\left(\frac{\partial U}{\partial R}+\frac{U}{R}\right)
$$

Once again the letter $\xi$ in Equation (10) is given instead of the parameters $\lambda, \mu, \gamma$, or $\eta$.

The elastic solution for the FG hollow cylinder is completed by the application of the initial and boundary conditions. The initial conditions can be expressed as

$$
T=\frac{\partial T}{\partial \tau}=0, U=\frac{\partial U}{\partial \tau}=0 \text { at } \tau=0
$$

The boundary conditions at the inner and outer radii of the FG cylinder may be expressed as

$$
\begin{array}{lll}
T=\mathrm{e}^{-\Omega \tau}, & \sigma_{11}=0 & \text { at } R=A, \\
T=0, & \sigma_{11}=0 & \text { at } R=1,
\end{array}
$$

where $\Omega$ is an exponent of the decayed heat flux.

\section{Numerical Scheme}

A finite element scheme is used here to get the temperature and radial displacement. The finite difference grids with spatial intervals $h$ (mesh width) in the radial direction and $k$ as the time step for the maximum time $\tau_{\text {max }}$, and use the subscripts $i$ and $n$ to denote the $i$ th discrete point in the $R$ direction and the $n$th discrete time. A mesh is defined by

$$
\begin{aligned}
& R_{i}=A+i h, \quad i=0,1,2, \cdots, N, \quad h=\frac{1-A}{N} . \\
& \tau^{n}=k n, \quad n=0,1,2,3, \cdots, K, \quad k=\frac{\tau_{\max }}{K} .
\end{aligned}
$$

The displacement and temperature may be given for positive integers $N$ and $K$ at any nodal location by

$$
U\left(R_{i}, \tau^{n}\right)=U_{i}^{n}, T\left(R_{i}, \tau^{n}\right)=T_{i}^{n} .
$$

The equation of motion and the heat conduction equation, given in Equations (7) and (8), may be expressed in the finite difference as

$$
\begin{aligned}
U_{i}^{n+1}= & f_{1} U_{i+1}^{n}+\left(2-f_{2}\right) U_{i}^{n}+f_{3} U_{i-1}^{n}-U_{i}^{n-1} \\
& -f_{4}\left(T_{i+1}^{n}-T_{i-1}^{n}\right)-f_{5} T_{i}^{n}, \\
T_{i}^{n+1}= & g_{1} T_{i+1}^{n}+2\left(1-g_{2}\right) T_{i}^{n}+g_{3} T_{i-1}^{n}-T_{i}^{n-1} \\
& -\frac{\varepsilon_{2}}{A+i h}\left(U_{i}^{n+1}-2 U_{i}^{n}+U_{i}^{n-1}\right) \\
& -\frac{\varepsilon_{2}}{2 h}\left(U_{i+1}^{n+1}-U_{i-1}^{n+1}-U_{i+1}^{n-1}+U_{i-1}^{n-1}\right),
\end{aligned}
$$

where

$$
f_{1}=\frac{k^{2}}{2 h \rho_{i}^{*}}\left[\beta_{i}^{*}\left(\frac{2}{h}+\frac{1}{A+i h}\right)+\left(\beta_{m}-\beta_{c}\right) V_{i}^{\prime}\right],
$$

$$
\begin{gathered}
f_{2}=\frac{k^{2}}{\rho_{i}^{*}}\left[\beta_{i}^{*}\left(\frac{2}{h^{2}}+\frac{1}{(A+i h)^{2}}\right)-\frac{\zeta}{A+i h} V_{i}^{\prime}\right] \\
f_{3}=\frac{k^{2}}{2 h \rho_{i}^{*}}\left[\beta_{i}^{*}\left(\frac{2}{h}-\frac{1}{A+i h}\right)-\left(\beta_{m}-\beta_{c}\right) V_{i}^{\prime}\right] \\
f_{4}=\frac{k^{2} \gamma_{i}^{*}}{2 h \rho_{i}^{*}}, \quad f_{5}=\frac{k^{2}\left(\gamma_{m}-\gamma_{c}\right)}{\rho_{i}^{*}} V_{i}^{\prime}, \\
\left\{g_{1}, g_{2}, g_{3}\right\}=\frac{k^{2} \varepsilon_{1}}{h^{2}}\left\{1+\frac{h}{2(A+i h)}, 1,1-\frac{h}{2(A+i h)}\right\}
\end{gathered}
$$

Note that

$\xi_{i}^{*}=\left(\xi_{m}-\xi_{c}\right) V_{i}+\xi_{c}, V_{i}=\left(\frac{A+i h-1}{A-1}\right)^{j}, i=0,1,2, \cdots, N$,

$V_{i}^{\prime}=\frac{j}{A-1}\left(\frac{A+i h-1}{A-1}\right)^{j-1}, i \neq 0, \quad i \neq N\left(V_{0}^{\prime}=V_{N}^{\prime}=0\right)$.

The mechanical stresses and electromagnetic stress are given, accordingly, by

$$
\begin{aligned}
& \sigma_{11}=\frac{\lambda_{i}^{*}+2 \mu_{i}^{*}}{2 k}\left(U_{i+1}^{n}-U_{i-1}^{n}\right)+\frac{\lambda_{i}^{*}}{A+i h} U_{i}^{n}-\gamma_{i}^{*} T_{i}^{n}, \\
& \sigma_{22}=\frac{\lambda_{i}^{*}+2 \mu_{i}^{*}}{2 k(A+i h)} U_{i}^{n}+\lambda_{i}^{*}\left(U_{i+1}^{n}-U_{i-1}^{n}\right)-\gamma_{i}^{*} T_{i}^{n}, \\
& \Sigma=\eta_{i}^{*}\left(\frac{U_{i+1}^{n}-U_{i-1}^{n}}{2 k}+\frac{U_{i}^{n}}{A+i h}\right) .
\end{aligned}
$$

The initial conditions in Equation (14) may be written as

$$
T_{i}^{0}=0, T_{i}^{-1}=T_{i}^{1}, \quad U_{i}^{0}=0, U_{i}^{-1}=U_{i}^{1} .
$$

Putting $n=0$ in Equations (18) and (19), one obtains

$$
\begin{aligned}
U_{i}^{1}= & \frac{1}{2}\left[f_{1} U_{i+1}^{0}+f_{3} U_{i-1}^{0}-f_{4}\left(T_{i+1}^{0}-T_{i-1}^{0}\right)\right], \\
T_{i}^{1}= & \frac{1}{2}\left(g_{1} T_{i+1}^{0}+g_{3} T_{i-1}^{0}\right)-\frac{\varepsilon_{2}}{A+i h} U_{i}^{1} \\
& -\frac{\varepsilon_{2}}{4 h}\left(U_{i+1}^{1}-U_{i-1}^{1}-U_{i+1}^{-1}+U_{i-1}^{-1}\right),
\end{aligned}
$$

where $i=1,2,3, \cdots, N-1$. The boundary conditions given in Equation (15) at $R=A$ may be written as

$$
T_{0}^{n}=\mathrm{e}^{-\Omega k n}, U_{-1}^{n}=U_{1}^{n}+\frac{2 h}{\lambda_{m}+2 \mu_{m}}\left(\frac{\lambda_{m}}{A} U_{0}^{n}-\gamma_{m} T_{0}^{n}\right),
$$

and at $R=1$, we get

$$
T_{N}^{n}=0, U_{N+1}^{n}=U_{N-1}^{n}-\frac{2 h}{\lambda_{c}+2 \mu_{c}}\left(\lambda_{c} U_{N}^{n}-\gamma_{c} T_{N}^{n}\right) .
$$


Putting $i=0$ in Equation (18) and using the forward difference approximation for the temperature's derivative with the aid of Equation (28), one obtains

$$
\begin{aligned}
U_{0}^{n+1}= & \left(f_{1}^{0}+f_{3}^{0}\right) U_{1}^{n}+\left(f_{2}^{0}+\frac{2 h}{A\left(\lambda_{m}+2 \mu_{m}\right)} f_{3}^{0}\right) U_{0}^{n}-U_{0}^{n-1} \\
& +f_{4}^{0}\left(T_{2}^{n}-4 T_{1}^{n}\right)-\left(\frac{2 h \bar{\gamma}_{m}}{\lambda_{m}+2 \mu_{m}} f_{3}^{0}-3 f_{4}^{0}\right) \mathrm{e}^{-\Omega k n},
\end{aligned}
$$

where

$$
\begin{aligned}
f_{1}^{0} & =\frac{k^{2} \beta_{m}}{2 h \rho_{m}}\left(\frac{2}{h}+\frac{1}{A}\right), \\
f_{2}^{0} & =2-\frac{k^{2} \beta_{m}}{\rho_{m}}\left(\frac{2}{h^{2}}+\frac{1}{A^{2}}\right), \\
f_{3}^{0} & =\frac{k^{2} \beta_{m}}{2 h \rho_{m}}\left(\frac{2}{h}-\frac{1}{A}\right), \\
f_{4}^{0} & =\frac{k^{2} \bar{\gamma}_{m}}{2 h \rho_{m}} .
\end{aligned}
$$

Putting $i=N$ in Equation (18) and using the backward difference approximation for the temperature's derivative with the aid of Equation (29), one obtains

$$
\begin{aligned}
U_{N}^{n+1}= & \left(f_{2}^{N}-\frac{2 h \lambda_{c}}{\lambda_{c}+2 \mu_{c}} f_{1}^{N}\right) U_{N}^{n}+\left(f_{1}^{N}+f_{3}^{N}\right) U_{N-1}^{n} \\
& -U_{N}^{n-1}-f_{4}^{N}\left(\bar{T}_{N-2}^{n}-4 \bar{T}_{N-1}^{n}\right),
\end{aligned}
$$

where

$$
\begin{aligned}
& f_{1}^{N}=\frac{k^{2} \beta_{c}(2+h)}{2 h^{2} \rho_{c}}, \\
& f_{2}^{N}=2-\frac{k^{2} \beta_{c}\left(2+h^{2}\right)}{h^{2} \rho_{c}}, \\
& f_{3}^{N}=\frac{k^{2} \beta_{c}(2-h)}{2 h^{2} \rho_{c}}, f_{4}^{N}=\frac{k^{2} \bar{\gamma}_{c}}{2 h \rho_{c}} .
\end{aligned}
$$

\section{Numerical Results}

The temperature, displacement and stresses for the present cylinder are obtained using the above finite element scheme. The results are presented in the non-dimensional form:

$$
u=U \times 10^{-6},\left(\sigma_{1}, \sigma_{2}\right)=\left(\sigma_{11}, \sigma_{22}\right) \times 10^{-8} .
$$

All results of this article are for aluminum as inner metal surface and alumina as outer ceramic surface. Generally, the magnetic permeability $\eta_{p}=\eta_{0} K_{p} \quad(p=m$ or $c$ ) is given in terms of the permeability of space $\eta_{0}=4 \pi \times 10^{-7} \mathrm{~N} / \mathrm{A}^{2}$ and the relative permeability for both aluminum $K_{m}$ and alumina $K_{c}$. The material properties are assumed to be as:

\section{Metal (aluminum):}

$$
\begin{gathered}
E_{m}=70 \mathrm{GPa}, v_{m}=0.35, \rho_{m}=2700 \mathrm{Kg} / \mathrm{m}^{3}, \\
\alpha_{m}=23.1 \times 10^{-6} / \mathrm{K}, K_{m}=2.3, \kappa_{m}=237 \mathrm{~W} /(\mathrm{m} \mathrm{K}) .
\end{gathered}
$$

Ceramic (alumina):

$$
\begin{gathered}
E_{c}=116 \mathrm{GPa}, \quad v_{c}=0.33, \quad \rho_{c}=3000 \mathrm{Kg} / \mathrm{m}^{3}, \\
\alpha_{c}=8.7 \times 10^{-6} / \mathrm{K}, \quad K_{c}=1.0, \quad \kappa_{m}=1.78 \mathrm{~W} /(\mathrm{m} \mathrm{K}) .
\end{gathered}
$$

Note that the properties of $\lambda, \mu$, and $\gamma$ for metal or ceramic are graded through the radial direction according to the following relations:

$$
\begin{aligned}
& \lambda_{p}=\frac{E_{p} v_{p}}{\left(1+v_{p}\right)\left(1-2 v_{p}\right)}, \mu_{p}=\frac{E_{p}}{2\left(1+v_{p}\right)}, \\
& \gamma_{p}=\left(3 \lambda_{p}+2 \mu_{p}\right) \alpha_{p},(p=m, c) .
\end{aligned}
$$

Results are presented in Figures 1-5 for temperature, radial displacement, radial stress, circumferential stress and electromagnetic stress according to the fixed constants

$b=1, A=0.25, T_{0}=27 \mathrm{~K}, H_{0}=2 \times 10^{5}$ Oersted, $\Omega=2.5$.

The sensitivity of the time parameter $\tau$ and the FGM exponent $j$ are discussed through the figures. Figure 1 represents the variation of the dimensionless temperature $T$ through the radial direction of the FGM annular cylinder. Four values of the time parameter $\tau$ with $j=3$ are used in Figure 1(a), while four values of the FGM exponent $j$ with $\tau=0.3$ are used in Figure 1(b). Similar results for the dimensionless displacement $u$, the radial stress $\sigma_{1}$, the circumferential stress $\sigma_{2}$ and the electromagnetic stress $\Sigma$ are plotted in Figures 2-5, respectively.

Figure 1 shows that the absolute value of the temperature decreases as the time parameter $\tau$ increases and the FGM exponent $j$ decreases. It is to be noted that the temperature is maximum for the homogeneous metal cylinders. The same behavior occurs for displacement and stress quantities. The solution satisfied the boundary conditions (see Figures 1 and $\mathbf{3}$ ) and the difference between homogeneous and FGM cases is shown. The variation of temperature, displacement, and stresses are due to the effect of inertia and magnetic field. It is seen that, the influence of the FGM on temperature, displacement and stresses is very pronounced. Finally, it is interested to see that all quantities my by vanished near and at the external ceramic surface of the annular cylinder. 


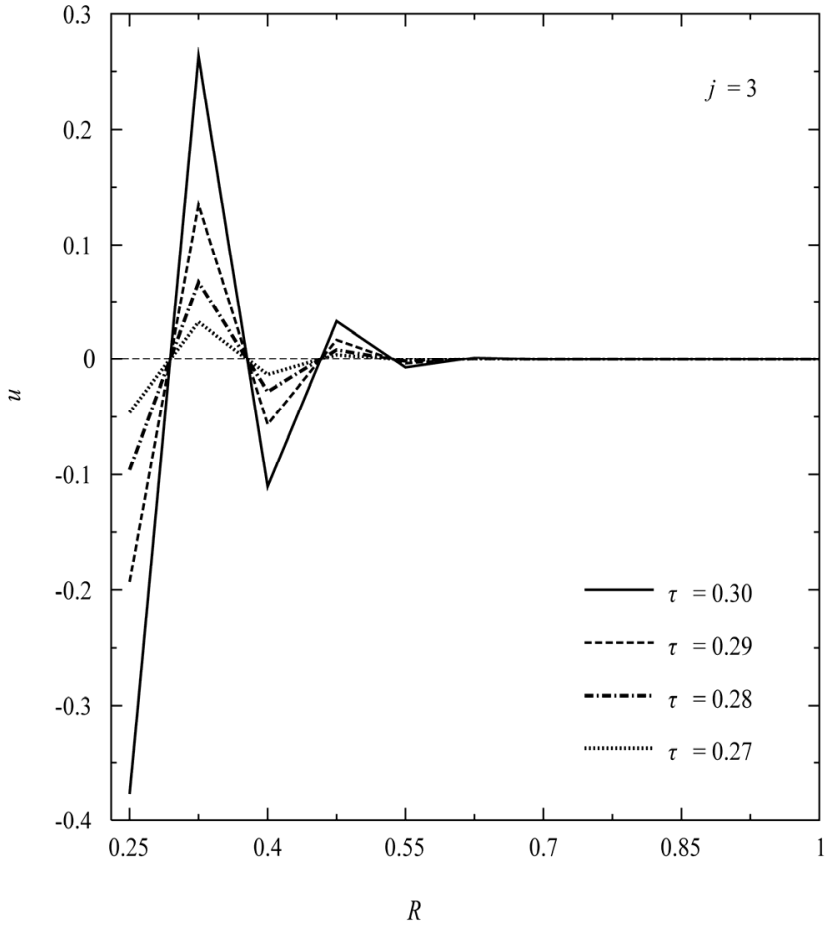

(a)

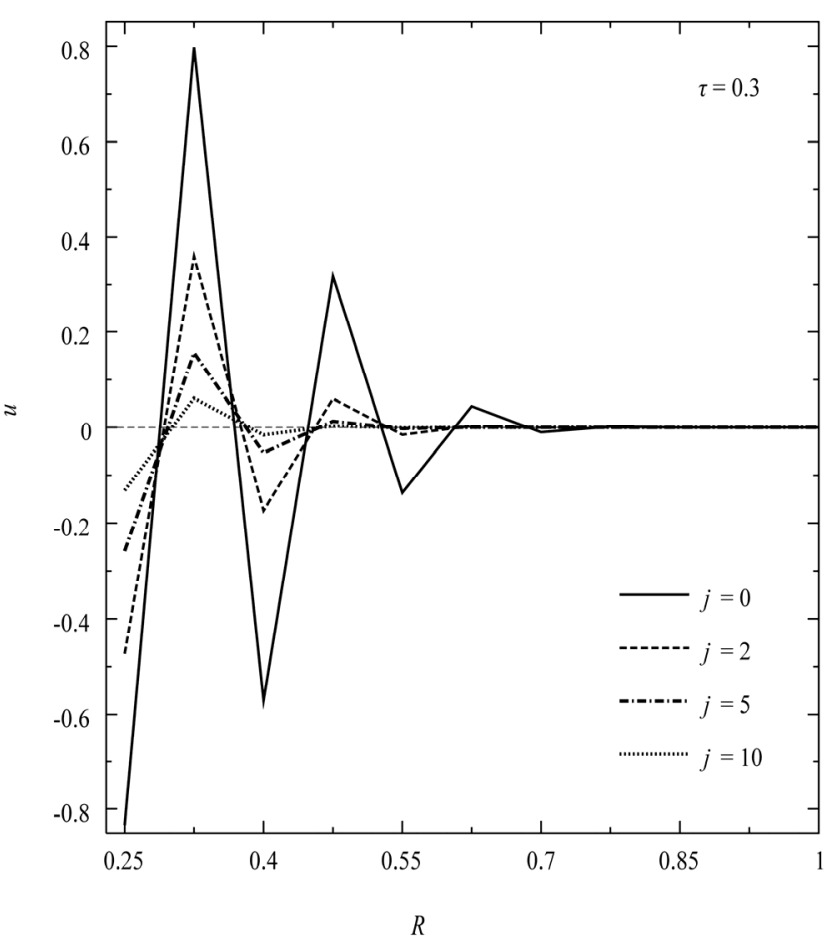

(b)

Figure 2. Displacement distribution through the radial direction of the FGM annular cylinder at (a) different times, and (b) for different values the gradation exponent.

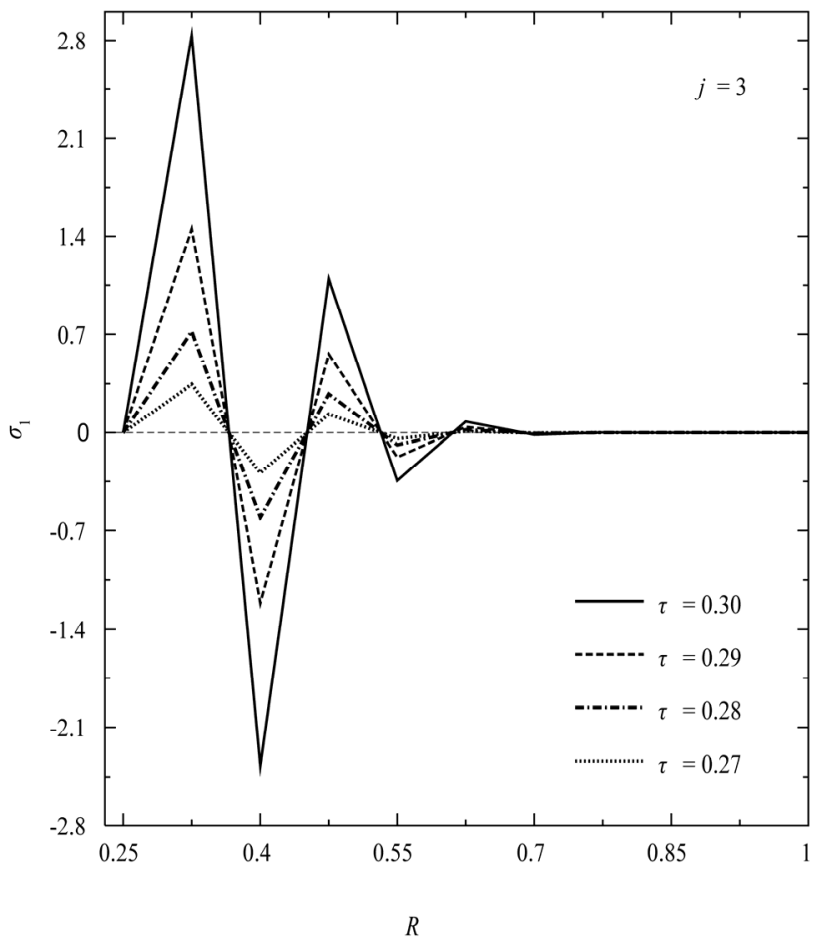

(a)

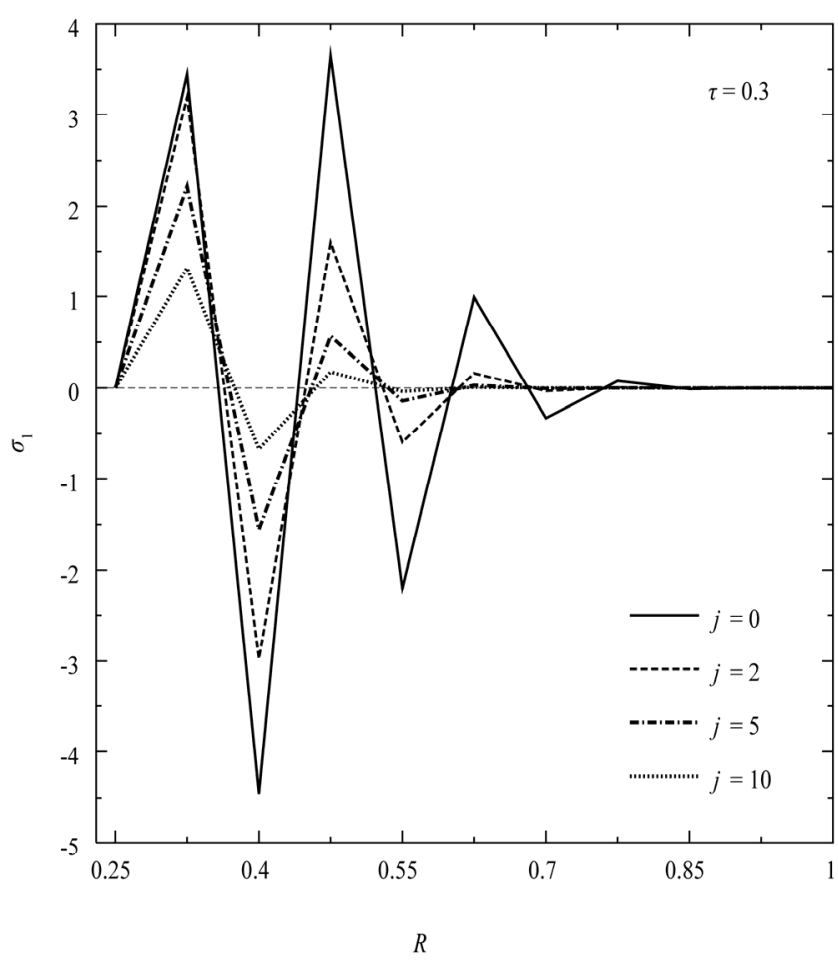

(b)

Figure 3. Radial stress distribution through the radial direction of the FGM annular cylinder at (a) different times, and (b) for different values the gradation exponent. 


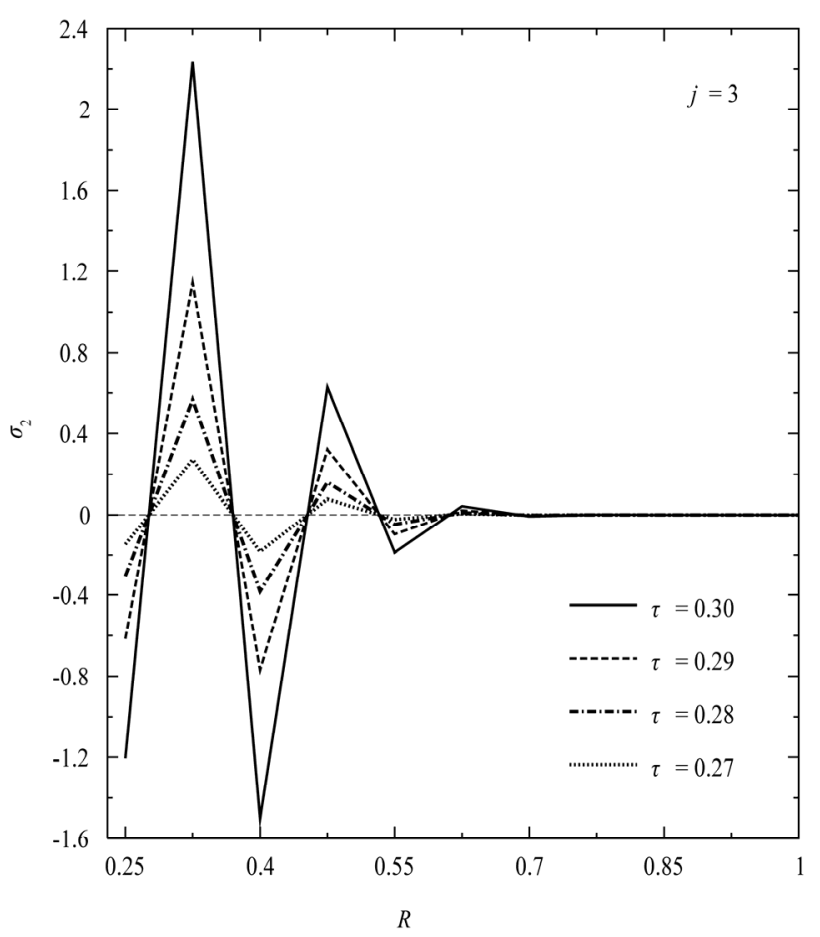

(a)

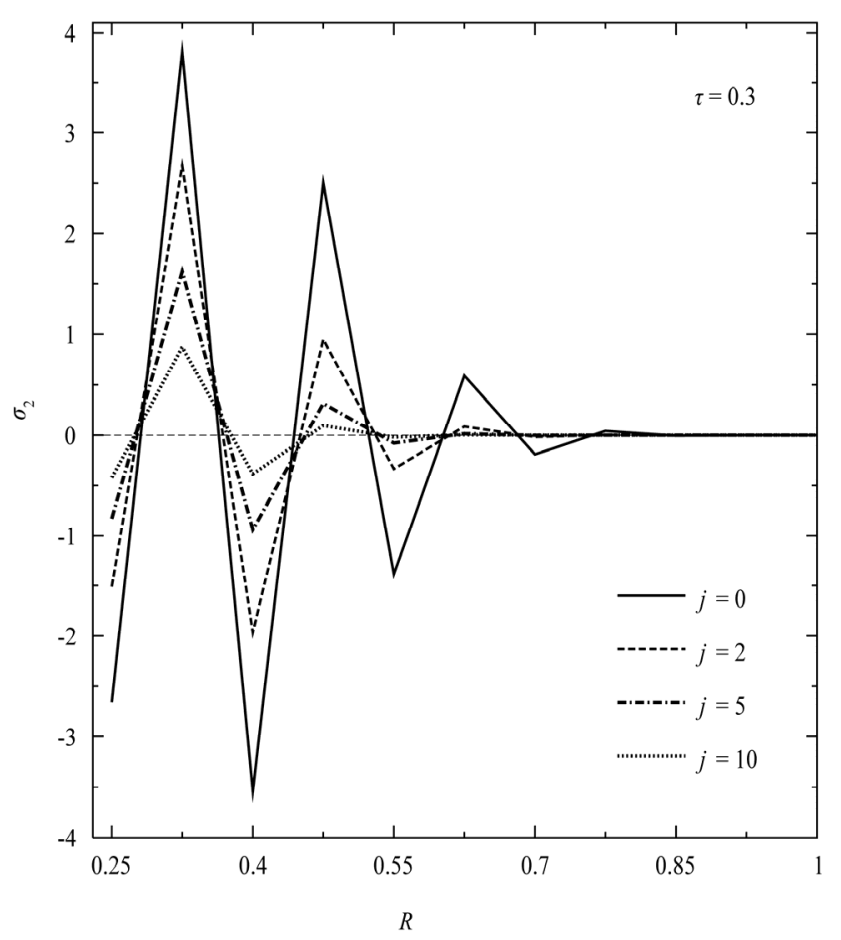

(b)

Figure 4. Circumferential stress distribution through the radial direction of the FGM annular cylinder at (a) different times, and (b) for different values the gradation exponent.

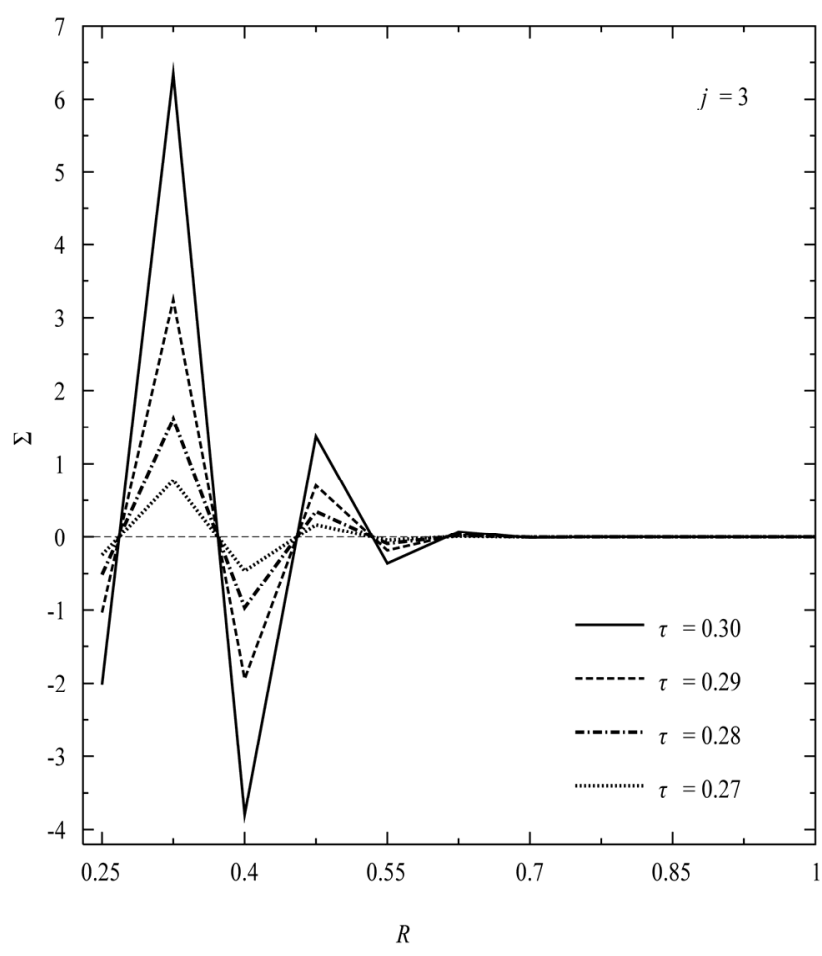

(a)

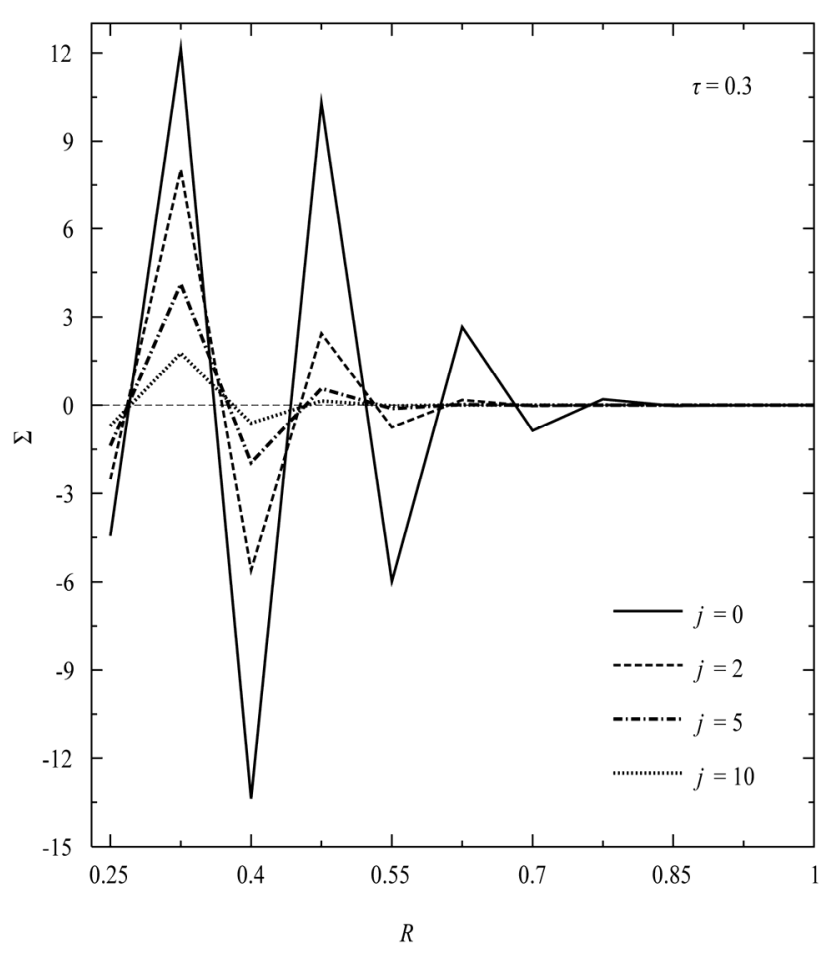

(b)

Figure 5. Electromagnetic stress distribution through the radial direction of the FGM annular cylinder at (a) different times, and (b) for different values the gradation exponent. 


\section{Conclusions}

The main contribution in this paper is to describe secondorder explicit finite-difference scheme. This scheme helps us to solve the coupled hyperbolic equations on a uniform grid, and is quite efficient for computation thermal stresses. The results obtained show that behavior of the temperature, displacement and stresses may change significantly by reason of influence of exponent heat flux and primary magnetic field in homogeneous and functionally graded cases. These results are specific for the example considered, but the example may have different trends because of the dependence of the results on the magnetic and thermal constants of the metal-ceramic functionally graded material.

\section{References}

[1] G. Paria, "Magneto-Elasticity and Magneto-Thermo-Elasticity," Advances in Applied Mechanics, Vol. 10, 1966, pp. 73-112. doi:10.1016/S0065-2156(08)70394-6

[2] X. Wang, G. Lu and S. R. Guillow, "Magnetothermodynamic Stress and Perturbation of Magnetic Field Vector in a Solid Cylinder," Journal of Thermal Stresses, Vol. 25, No. 10, 2002, pp. 909-926. doi:10.1080/0149573029 0074397

[3] X. Wang and H. L. Dai, "Magnetothermodynamic Stress and Perturbation of Magnetic Field Vector in an Orthotropic Thermoelastic Cylinder," International Journal of Engineering Science, Vol. 42, No. 5-6, 2004, pp. 539556. doi:10.1016/j.ijengsci.2003.08.002

[4] X. Wang and K. Dong, "Magnetothermodynamic Stress and Perturbation of Magnetic Field Vector in a NonHomogeneous Thermoelastic Cylinder," European Journal of Mechanics-A/Solids, Vol. 25, No. 1, 2006, pp. 98109. doi:10.1016/j.euromechsol.2005.07.003

[5] S. Banerjee and S. K. Roychoudhuri, "Magneto-ThermoElastic Interactions in an Infinite Isotropic Elastic Cylinder Subjected to a Periodic Loading," International Journal of Engineering Science, Vol. 35, No. 4, 1997, pp. 437- 444. doi:10.1016/S0020-7225(96)00070-5

[6] H. L. Dai and Y. M. Fu, "Magnetothermoelastic Interactions in Hollow Structures of Functionally Graded Material Subjected to Mechanical Loads," International Journal of Pressure Vessels and Piping, Vol. 84, No. 3, 2007, pp. 132-138. doi:10.1016/j.ijpvp.2006.10.001

[7] P. F. Hou and A. Y. T. Leung, "The Transient Responses of Magneto-Electro-Elastic Hollow Cylinders," Smart Materials and Structures, Vol. 13, No. 4, 2004, pp. 762776. doi:10.1088/0964-1726/13/4/014
[8] G. R. Buchanan, "Free Vibration of an Infinite Magneto-Electroelastic Cylinder,” Journal of Sound and Vibration, Vol. 268, No. 2, 2003, pp. 413-426. doi:10. 1016/S0022-460X(03)00357-2

[9] A. R. Annigeri, N. Ganesan and S. Swarnamani, "Free Vibrations of Clamped-Clamped Magneto-Electro-Elastic Cylindrical Shells,” Journal of Sound and Vibration, Vol. 292, No. 1-2, 2006, pp. 300-314. doi:10.1016/j.jsv.2005. 07.043

[10] P. F. Hou, H. J. Ding and A. Y. T. Leung, "The Transient Responses of a Special Non-Homogeneous MagnetoElectro-Elastic Hollow Cylinder for Axisymmetric Plane Strain Problem,” Journal of Sound and Vibration, Vol. 291, No. 1-2, 2006, pp. 19-47. doi:10.1016/j.jsv.2005. 05.022

[11] M. R. Abd-El-Salam, A. M. Abd-Alla and H. A. Hosham, "A Numerical Solution of Magneto-Thermoelastic Problem in Non-Homogeneous Isotropic Cylinder by the FiniteDifference Method,” Applied Mathematical Modelling, Vol. 31, No. 8, 2007, pp. 1662-1670. doi:10.1016/j.apm. 2006.05.009

[12] M. Higuchi, R. Kawamura and Y. Tanigawa, "Dynamic and Quasi-Static Behaviors of Magneto-Thermo-Elastic Stresses in a Conducting Hollow Circular Cylinder Subjected to an Arbitrary Variation of Magnetic Field," International Journal of Mechanical Sciences, Vol. 50, No. 3, 2008, pp. 365-379.

[13] R. K. Bhangale and N. Ganesan, "Free Vibration Studies of Simply Supported Non-Homogeneous Functionally Graded Magneto-Electro-Elastic Finite Cylindrical Shells," Journal of Sound and Vibration, Vol. 288, No. 1-2, 2005 , pp. 412-422. doi:10.1016/j.jsv.2005.04.008

[14] T. Kong, D. X. Li and X. Wang, "Thermo-MagnetoDynamic Stresses and Perturbation of Magnetic Field Vector in a Non-Homogeneous Hollow Cylinder," Applied Mathematical Modelling, Vol. 33, No. 7, 2009, pp. 2939-2950. doi:10.1016/j.apm.2008.10.003

[15] X. Wang and H. L. Dai, "Magneto-Thermo-Dynamic Stress and Perturbation of Magnetic Field Vector in a Hollow Cylinder," Journal of Thermal Stresses, Vol. 3, 2004, pp. 269-288. doi:10.1080/01495730490423900

[16] H. L. Dai and X. Wang, "Magneto-Thermo-ElectroElastic Transient Response in a Piezoelectric Hollow Cylinder Subjected to Complex Loadings," International Journal of Solids and Structures, Vol. 43, No. 18-19, 2006, pp. 5628-5646. doi:10.1016/j.ijsolstr.2005.06.092

[17] S. B. Mukhopadhyay, "Thermoelastic Interactions without Energy Dissipation in an Unbounded Body with a Spherical Cavity Subjected to Harmonically Varying Temperature,” Mechanics Research Communications, Vol. 31, No. 1, 2004, pp. 81-89. doi:10.1016/S0093-6413(03) 00082-X 